Research Paper

\title{
Anti-inflammatory effects of Flos Lonicerae Japonicae Water Extract are regulated by the STAT/NF-KB pathway and HO-1 expression in Virus-infected RAW264.7 cells
}

\author{
Hui-Wen Linn',2\#, Yi-Ju Lee ${ }^{3 \#, ~ D e n g-J y e ~ Y a n g ~} 4$, Ming-Chang Hsieh ${ }^{5}$, Ching-Chung Chen ${ }^{1}$, Wei-Li Hsu 6 , \\ Yuan-Yen Chang ${ }^{\boxplus 凶}$ and Cheng-Wei Liu ${ }^{8}$ \\ 1. Department of Optometry, Asia University, Taichung, Taiwan. \\ 2. Genetics Center, Department of Medical Research, China Medical University Hospital, and School of Chinese Medicine, China Medical University, \\ Taichung, Taiwan. \\ 3. Department of Pathology, Chung-Shan Medical University; Department of Pathology, Chung-Shan Medical University Hospital, Taichung, Taiwan \\ 4. Institute of Food Safety and Health Risk Assessment, National Yang-Ming University, Taipei, Taiwan. \\ 5. Department of Medical Laboratory and Biotechnology, Chung Shan Medical University; Clinical Laboratory, Chung Shan Medical University Hospital, \\ Taichung, Taiwan. \\ 6. Graduate Institute of Microbiology and Public Health, College of Veterinary Medicine, National Chung Hsing University, Taichung, Taiwan \\ 7. Department of Microbiology and Immunology, School of Medicine, and Chung Shan Medical University; Clinical Laboratory, Chung Shan Medical \\ University Hospital, Taichung, Taiwan. \\ 8. Department of Post-Modern Agriculture, MingDao University, Changhua, Taiwan. \\ \#Equal contributions as first authors.
}

$\square$ Corresponding authors: Dr. Cheng-Wei Liu \& Yuan-Yen Chang contributed equally to this work. Tel.: +886-4-24730022 ext. 12028; Fax: +886-4-24727178; E-mail: chad888@mdu.edu.tw, cyy0709@csmu.edu.tw.

(c) The author(s). This is an open access article distributed under the terms of the Creative Commons Attribution License (https://creativecommons.org/licenses/by/4.0/). See http://ivyspring.com/terms for full terms and conditions.

Received: 2020.11.20; Accepted: 2021.03.18; Published: 2021.03.30

\begin{abstract}
This study examined the effect of the Flos Lonicerae Japonicae water extract (FLJWE), chlorogenic acid, and luteolin on pseudorabies virus (PRV)-induced inflammation in RAW264.7 cells and elucidated related molecular mechanisms. The results revealed that FLJWE and luteolin, but not chlorogenic acid, inhibited the production of inducible nitric oxide synthase (iNOS), cyclooxygenase-2 (COX-2), and inflammatory cytokines in PRV-infected RAW 264.7 cells. We found that the FLJWE and luteolin suppressed nuclear factor (NF)-KB activation by inhibiting the phosphorylation of signal transducer and activator of transcription 1 and 3 (STATI and STAT3, respectively). Moreover, the FLJWE significantly upregulated the expression of $\mathrm{pNrf} 2$ and its downstream target gene heme oxygenase-1 (HO-1). Our data indicated that FLJWE and luteolin reduced the expression of proinflammatory mediators and inflammatory cytokines, such as COX-2 and iNOS, through the suppression of the JAK/STAT1/3-dependent NF-KB pathway and the induction of HO-1 expression in PRV-infected RAW264.7 cells. The findings indicate that the FLJWE can be used as a potential antiviral agent.
\end{abstract}

Key words: Flos Lonicerae Japonicae water extract (FLJWE), pseudorabies virus (PRV), antiviral inflammatory, heme oxygenase-1 (HO-1)

\section{Introduction}

Flos Lonicerae Japonicae (FLJ), also commonly referred to as honeysuckle, is the flower bud of Lonicera japonica Thunb. (Caprifoliaceae) [1]. FLJ has long been used in Chinese herbal medicine because of its antibacterial, antiviral, anti-inflammatory, antioxidative, and hepatoprotective properties [2-5], which may be associated with its active components such as organic acids, flavones, and saponins).
FLJ is a traditional herbal medicine that has been widely used in East Asia as an anti-inflammatory and antioxidative agent. Studies have reported that the FLJ extract exerted a strong anti-inflammatory effect and could effectively inhibit bacterial lipopolysaccharide (LPS)-induced inflammatory mediators in vitro $[4,6]$. In addition, these studies have suggested that FLJ could inhibit the responses of various 
inflammatory factors, such as cyclooxygenase- 2 (COX-2), tumor necrosis factor-a (TNF-a), and interleukin-6 (IL-6), by regulating the phosphorylation of phosphoinositide 3-kinase (PI3K)/Akt and mitogen-activated protein kinases (MAPKs).

In the present study, we evaluated whether organic FLJ flowers, which are suited to the local environment of Taiwan, would be beneficial in producing a pollutant-free, high-quality FLJ water extract (FLJWE). These plants were grown and subjected to quality control according to the guidelines of organic farming and the global good agriculture practice (GAP) system. In addition, we analyzed the composition and biological activity of the FLJWE.

Our previous studies have reported that RAW264.7 cells (macrophage-like cell line from a mouse) could be used as an in vitro screening platform for pseudorabies virus (PRV)-induced inflammation [7-10]. The main aim of this study was to investigate the anti-inflammatory effect of the FLJWE on virusinduced inflammation.

Inflammation at the site of injury or infection is one of the earliest biological functions suggested to be mediated by the phosphorylation of STAT proteins [11-14]. The preferential activation of signal transducer and activator of transcription 1 and 3 (STAT1 and STAT3, respectively) was observed in response to the release of endogenous IL-6 within stimulated cells $[15,16]$. However, the molecular mechanisms underlying the anti-inflammatory effect of the FLJWE on virus-induced inflammation remain unclear.

Heme oxygenase-1 (HO-1), an inducible, anti-inflammatory, and immunosuppressive enzyme, could protect against apoptosis, inflammation, and oxidative stress in vitro $[17,18]$. A study reported that HO-1 attenuated inflammation and modulated immune responses both in vitro and in vivo [19]. Nuclear factor erythroid-2-related factor 2 (Nrf2) is the main transcription factor that induces the expression of HO-1 and other antioxidant enzymes $[20,21]$. Therefore, in this study, we elucidated the mechanism through which the FLJWE resulted in the expression of HO-1 and pNrf-2 (Nrf2 activation) in PRV-infected RAW264.7 cells.

Phenolics and flavonoids play key roles in the antioxidative activity of plants [22-25]. In this study, we first evaluated the antioxidative and antiinflammatory properties of the FLJWE by examining their radical scavenging activity and total phenolic concentration. Furthermore, we examined whether the anti-inflammatory effect was induced by the production of inducible nitric oxide synthase (iNOS),
COX-2, and inflammatory cytokines, such as IL-6, TNF- $\alpha$, RANTES, and monocyte chemoattractant protein-1 (MCP-1), through the inactivation of NF- $\mathrm{KB}$ and STAT1/3 and the activation of HO-1 in PRVinfected RAW264.7 macrophages.

\section{Materials and methods}

\section{Chemicals and reagents}

Potassium hydroxide $(\mathrm{KOH})$ was purchased from Merck (Darmstadt, Germany). The following reagents and chemicals were procured from Sigma (St. Louis, MO, USA): glutamine, 3-(4,5-dimethylthiazol-2-yl)-2, penicillin, streptomycin, phosphatebuffered saline, ethylenediaminetetraacetic acid, phenylmethanesulphonyl fluoride, sodium chloride, Tris, and Triton X-100. Fetal bovine serum (FBS) and RPMI-1640 medium were purchased from Gibco/ Invitrogen (Carlsbad, CA, USA). In addition, specific antibodies for COX-2, iNOS, NF-KB, and $\beta$-actin were obtained from Santa Cruz Biotechnology (Santa Cruz, CA, USA). Enzyme-linked immunosorbent assay (ELISA) kits used to measure the levels of TNF- $a$, IL-6, MCP-1, and RANTES were procured from R\&D Systems (Minneapolis, MN, USA). Horseradish peroxidase-conjugated antigoat, antirabbit, and mouse immunoglobulin $\mathrm{G}$ were purchased from BioRad Laboratories (Hercules, CA, USA). Antibodies against STAT1/3 and pSTAT1/3 were obtained from Cell Signaling Technology (Danvers, MA, USA).

\section{Preparation of the FLJWE}

L. japonica Thunb. was planted in the experimental farm of Ming Dao University (Changhua, Taiwan). Organic farming methods were used to manage and cultivate the plants. Plant production and quality control were in accordance with organic farming and global GAP protocols. We harvested flower buds at different stages from July to September in 2014. The concentrations of 398 pesticides (Appendix) in FLJ were analyzed using an AOAC Official Method of Analysis (SGS Taiwan Ltd.). We determined that the analyzed samples were free from the 398 pesticides. A total of $500 \mathrm{~g}$ of samples was collected for each group according to the developmental characteristics of the FLJ flower and then dried at $40^{\circ} \mathrm{C}$ for $96 \mathrm{~h}$ until fully dehydration. They were then ground into fine powder, which was stored in a cabinet drier until use in further experiments. Using water as a solvents, we obtained $15 \mathrm{~g} / \mathrm{L}$ of the FLJWE by steeping a suitable amount of dried FLJ in boiled distilled water $\left(100^{\circ} \mathrm{C}\right)$ for $30 \mathrm{~min}$ before filtration (ADVANTEC No. 1). The water extract samples were stored at $-20^{\circ} \mathrm{C}$. 


\section{Total polyphenolic profile of the FLJWE}

We spectrophotometrically examined the concentrations of total flavonoids and phenolic compounds in the FLJWE following a previous method [9,11]. Phenolic acid compounds, namely gallic acid, gentisic acid, chlorogenic acid, p-hydroxybenzoic acid, vanillic acid, caffeic acid, p-coumaric acid, ferulic acid, sinapic acid, syringic acid, p-anisic acid, and rosmarinic acid, and flavonoid standards, namely those of catechin, rutein, quercitrin, luteolin, rosmaric acid, neohesperidin, hesperidin, morin, eriodictyol, daidzein, glycitrin, quercetin, diosmin, naringenin, genistein, nesperetin, apigenin, kaempferol, and isorhamnetin, were purchased from Sigma. The aforementioned phenolic acids and flavonoid compounds in the FLJWE were identified through high-performance liquid chromatography (HPLC) based on UV absorbance and retention time and quantified using the standard curves of authentic compounds.

\section{Cell line and virus}

Murine macrophage RAW264.7 cells were obtained from the American Type Culture Collection (Manassas, VA, USA). These cells were cultured in RPMI-1640 medium supplemented with antibiotics (100 units $/ \mathrm{mL}$ of penicillin and $100 \mu \mathrm{g} / \mathrm{mL}$ of streptomycin), $2 \mathrm{mM}$ glutamine, and $10 \%$ heatinactivated FBS in a humidified incubator (Astek Co. Fukuoka, Japan) containing $5 \% \mathrm{CO}_{2}$ at $37^{\circ} \mathrm{C}$. The stock of PRV (strain of TNL) used in this study was amplified from PK15 cells; in addition, the titer was determined by performing a standard plaque assay in PK15 cells, as previously described $[9,10]$.

\section{Cell viability assay}

RAW264.7 cells were seeded into 24-well plates at $1 \times 10^{6}$ cells/well and incubated for $24 \mathrm{~h}$; these cells were subjected to a 1.5 - $\mathrm{h}$ preincubation process at different FLJWE concentrations $(2.5,5,10,20$, and 40 $\mu \mathrm{g} / \mathrm{mL}$ ). Cell viability was measured using blue formazan, the metabolized product of 3-(4, 5-dimethylthiazol-2-yl)-2, 5-diphenyltetrazolium bromide) (MTT); blue formazan is activated by mitochondrial dehydrogenases only in live cells [9, 10].

\section{Determination of proinflammatory cytokines}

RAW264.7 cells were pretreated under various conditions for $24 \mathrm{~h}$. ELISA was performed to measure the levels of RANTES, IL-6, TNF- $a$ and MCP-1 in the culture medium (supernatant) according to manufacturer protocols $[9,10]$.

\section{Western blotting}

RAW264.7 cells were pretreated under different conditions for $24 \mathrm{~h}$. After the incubation of cell lysates with specific antibodies, immunoblotting was performed, as described previously $[9,10]$.

\section{Statistical analysis}

All experiments were performed in triplicate, and mean values were calculated. Analysis of variance was used to evaluate data, and differences in the mean \pm standard deviation were determined using Duncan's multiple range test. The significance level was set at $p<0.05$.

\section{Results and discussion}

\section{Polyphenolic profile of the FLJWE}

The concentrations of phenolic acids and flavonoid compounds in the FLJWE were determined through HPLC (Fig. 1A). The concentrations of phenolic acids and total flavonoids in the FLJWE were approximately $41.27 \pm 2.42$ and $37.19 \pm 2.91 \mathrm{mg}$ per $100 \mathrm{~mL}$ of extract, respectively (Fig. 1B). Previous studies have identified the bioactive markers of chlorogenic acid and luteolin in FLJ $[26,27]$. The concentrations of chlorogenic acid and luteolin were $37.12 \pm 2.18$ and $8.78 \pm 0.93 \mathrm{mg}$ per $100 \mathrm{~mL}$ of extract, respectively. Furthermore, the concentrations of p-hydroxybenzoic acid, epicatechin, hesperidin, and morin were $4.15 \pm 0.24,10.24 \pm 0.56,6.92 \pm 0.73$, and $11.25 \pm 0.69 \mathrm{mg}$ per $100 \mathrm{~mL}$ of extract, respectively (Fig. 1B). These findings indicated that among phenolic acids, the proportion of chlorogenic acid $(89.94 \%)$ was found to be the highest in the FLJWE. Phenolics and flavonoids play key roles in the antioxidative activity of plants [22-23, 28]. In addition, chlorogenic acid and luteolin could activate Nrf2 in in vitro models, resulting in the upregulation of HO-1 and the attenuation of excessive inflammatory responses [20, 29]. Moreover, recent studies have indicated that epicatechin [30], hesperidin [31], and morin [32] exerted an anti-inflammatory effect on LPS-stimulated cells. On the basis of the antioxidative and anti-inflammatory properties of the major phenolic acids and flavonoid compounds, we investigated the in vitro effect of the FLJWE on virus-induced inflammation.

\section{Cytotoxicity of the FLJWE in RAW264.7 cells}

We performed an MTT assay to examine the cytotoxic effects of the FLJWE at concentrations ranging from 2.5 to $40 \mu \mathrm{g} / \mathrm{mL}$ on the viability of RAW264.7 cells. The results revealed that an FLJWE concentration of $<10 \mu \mathrm{g} / \mathrm{mL}$ exerted no toxic effects ( $p$ $>0.05$ ) on RAW264.7 cells (Fig. 2). Therefore, we used 
the FLJWE concentrations of $2.5,5$ and $10 \mu \mathrm{g} / \mathrm{mL}$ in subsequent experiments.

\section{Effect of the FLJWE on proinflammatory cytokine production in RAW264.7 cells infected with PRV}

The proinflammatory cytokines RANTES, MCP-1, IL-6, and TNF-a are involved in the immunopathology of acute or chronic inflammatory diseases $[4,9,33]$. Therefore, we investigated the effect of the FLJWE on proinflammatory cytokine secretion in PRV-infected RAW264.7 cells. The cells were preincubated in the presence or absence of the FLJWE for $1.5 \mathrm{~h}$ before PRV infection (at a multiplicity of infection of 0.1 ). The cytokine concentration was measured using a commercially available ELISA kit. The results revealed that the FLJWE dosedependently suppressed $(p<0.05)$ the levels of TNF- $\alpha$, IL-6, RANTES, and MCP-1 secreted in PRV-infected RAW264.7 cells (Fig. 3).

\section{Effect of the FLJWE on NF-KB, COX-2, and iNOS expression in RAW264.7 cells infected with PRV}

Our previous study revealed that PRV infection activates signal transduction, including NF- $\mathrm{KB}$ and MAPK cascades [8-10]. NF- $\mathrm{KB}$ is a well-known transcription factor that induces the expression of

A.

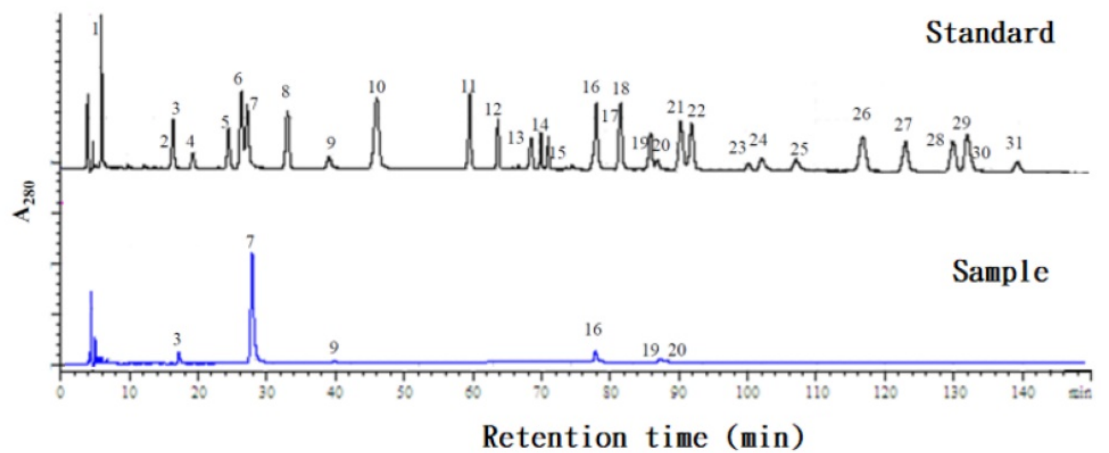

B.

\begin{tabular}{|l|l|l|}
\hline $\begin{array}{c}\text { Peak } \\
\text { no. }\end{array}$ & \multicolumn{1}{|c|}{ Compound } & \multicolumn{1}{c|}{$\begin{array}{c}\text { Contents * } \\
(\mathrm{mg} / 100 \mathrm{ml})\end{array}$} \\
\hline 3 & p-Hydroxybenzic acid & $4.15 \pm 0.24$ \\
\hline 7 & Chlorogenic acid & $37.12 \pm 2.18$ \\
\hline 9 & Epicatechin & $10.24 \pm 0.56$ \\
\hline 16 & Luteolin & $8.78 \pm 0.93$ \\
\hline 19 & Heaperidin & $6.92 \pm 0.73$ \\
\hline 20 & Morin & $11.25 \pm 0.69$ \\
\hline Phenolic acid amount & $41.27 \pm 2.42$ \\
\hline Flavonoid amount & $37.19 \pm 2.91$ \\
\hline Total amount & $78.46 \pm 5.33$ \\
\hline
\end{tabular}

Figure 1. HPLC chromatograms of standards and the FLJWE. Peaks: (1) gallic acid, (2) gentisic acid, (3) p-hydroxybenzoic acid, (4) catechin, (5) vanillic acid, (6) caffeic acid, (7) chlorogenic acid, (8) syringic acid, (9) epicatechin, (10) p-coumaric acid, (11) ferulic acid, (12) sinapic acid, (13) p-anisic acid, (14) rutein, (15) quercitrin, (16) luteolin, (17) rosmaric acid, (18) neohesperidin, (19) hesperidin, (20) morin, (21) eriodictyol, (22) daidzein, (23) glycitrin, (24) quercetin, (25) diosmin, (26) naringenin (27) genistein, (28) nesperetin, (29) apigenin, (30) kaempferol, and (31) isorhamnetin. Data are presented as mean \pm standard error of mean $(n=3)$. proinflammatory genes such as iNOS and COX-2. To confirm that the FLJWE exerted an inflammatory effect on virus-induced inflammation, the expression of inflammation-related proteins, namely iNOS, COX2, and NF-kB, was examined through Western blotting. In the experiment, RAW264.7 cells were either infected with PRV or not. The expression of iNOS, COX2, and NF-kB proteins was significantly increased in the PRV-infected group compared with the uninfected group and was significantly suppressed by the FLJWE at a concentration of 10 $\mu \mathrm{g} / \mathrm{mL}$. The FLJWE dose-dependently inhibited the PRV-induced expression of NF-kB, COX-2, and iNOS (Fig. 4). These findings indicated that the FLJWE might exert an anti-inflammatory effect on virus-induced inflammation.

\section{Effect of the FLJWE on STAT1 and STAT3 expression in RAW264.7 cells infected with PRV}

Recent studies have indicated that LPS induces IL-6 production by activating STAT1 and STAT3 signaling pathways $[9,33]$. Phosphorylated STAT dimers are major transcription factors that lead to inflammatory responses. Our previous study indicated that luteolin attenuated inflammatory responses through the suppression of the STAT1/3dependent NF-KB pathway in PRV-infected RAW264.7 cells [10]. Therefore, we examined whether the FLJWE can modulate PRV-induced STAT1 and STAT3 activation. As shown in Fig. 5, PRV infection considerably increased STAT1 and STAT3 phosphorylation compared with in the control condition. However, pretreatment with the FLJWE dose-dependently reduced PRV-induced STAT1 and STAT3 phosphorylation.

\section{Effect of the FLJWE on the expression of HO-1 and pNrf2 in PRV-infected RAW264.7 cells}

HO-1 is a member of the heat-shock protein family; it is a crucial antioxidant and antiinflammatory protein and is regulated by the activation of the major transcription factor Nrf2 [34]. HO-1 and its end products possess antioxidative, antiinflammatory, antiapoptotic, and 
antiproliferative [35] and antiviral [36, 37] properties. Therefore, we evaluated whether the FLJWE exerts anti-inflammatory effects by inducing the expression of HO-1 and pNrf2 (Nrf2 activation). As shown in Fig. 6, PRV infection considerably reduced the phosphorylation of Nrf2 (pNrf2) compared with in the control condition. However, compared with the positive control (PRV-infected group), pretreatment with the FLJWE upregulated the protein levels of pNrf2 and HO-1. These results suggest that the FLJWE induces HO-1 in an Nrf2-dependent manner.

\section{Effect of the FLJWE and a comparison of the effects of luteolin and chlorogenic acid on proinflammatory cytokine production in PRV-infected RAW264.7 cells}

Previous studies have examined the concentrations of chlorogenic acid and luteolin as standard chemicals to evaluate the chemical activity of FLJ [4, 38]. Therefore, we investigated the anti-inflammatory effects of the FLJWE, chlorogenic acid, and luteolin on infected RAW264.7 cells. The cells were preincubated in the presence or absence of the FLJWE, chlorogenic acid $(250 \mu \mathrm{M})$, and luteolin
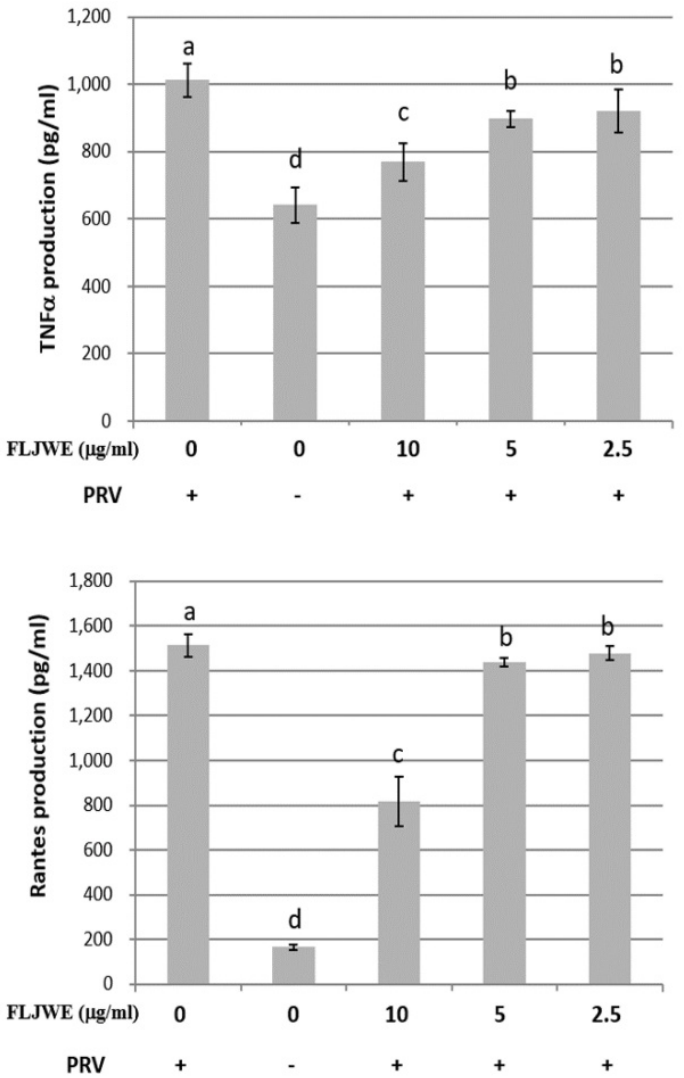

$(10 \mu \mathrm{M})$ for $1.5 \mathrm{~h}$ before PRV infection. The cytokine concentration was measured using a commercially available ELISA kit. The findings indicated that the FLJWE and luteolin considerably inhibited the production of TNF-a, IL-6, MCP-1, and RANTES in infected RAW264.7 cells. However, chlorogenic acid did not effectively inhibit the production of various cytokines (Fig. 7).

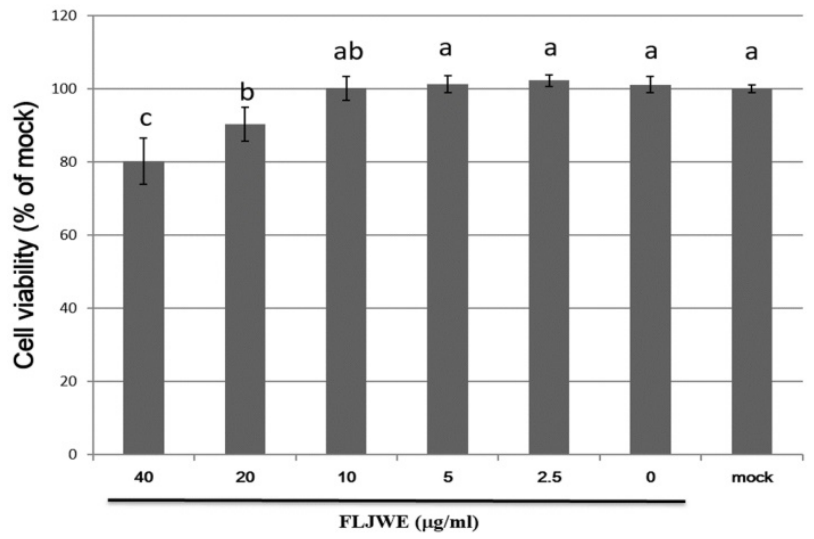

Figure 2. Cell viability of RAW264.7 cells treated with various concentrations of the FLJWE. RAW264.7 cells were treated with various concentrations of FLJWE, and their cell viability was examined using an MTT assay. Data are presented as mean \pm SD $(n=3)$. Mean values with different letters are significantly different $(p<0.05)$.
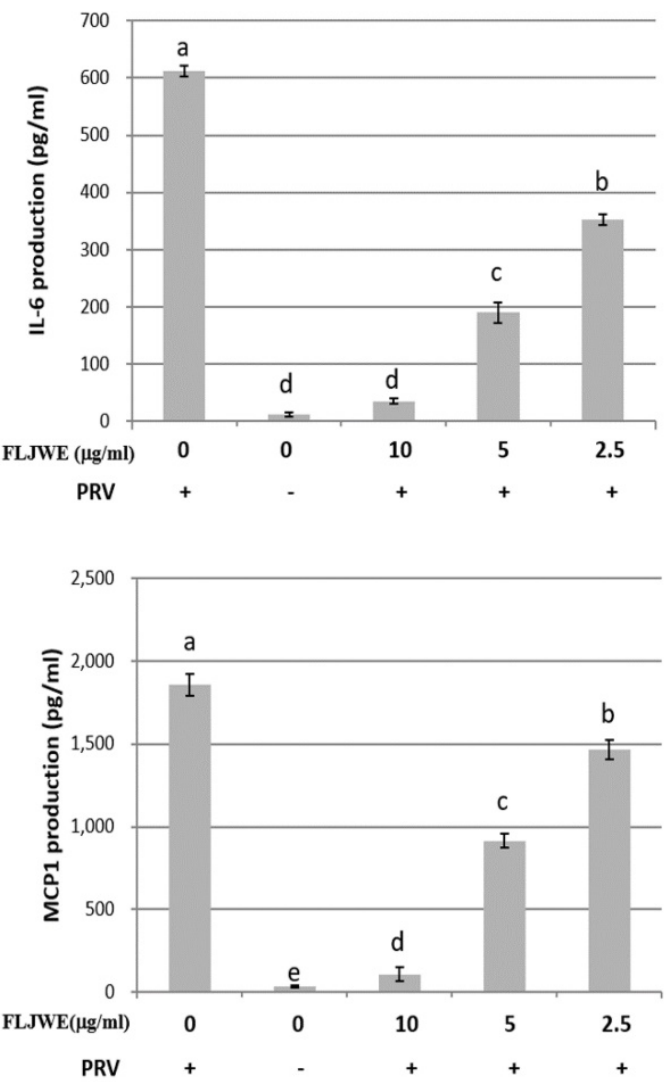

Figure 3. Inhibitory effects of the FLJWE on IL-6, MCP-1, RANTES, and TNF- $\alpha$ production in PRV-infected RAW264.7 cells. RAW264.7 cells were not treated or pretreated with various concentrations of the FLJWE $(10,5$, and $2.5 \mu \mathrm{g} / \mathrm{mL}$ ) for $1.5 \mathrm{~h}$ and then infected or not infected (control) with PRV (at an MOI of 0.1$)$. After $24 \mathrm{~h}$, the cultured medium was assayed to determine the levels of TNF- $\alpha$, IL-6, RANTES, and MCP-1 through an enzyme-linked immunosorbent assay. The results are presented as mean \pm SD ( $n=3)$. Mean values with different letters are significantly different $(p<0.05)$. 


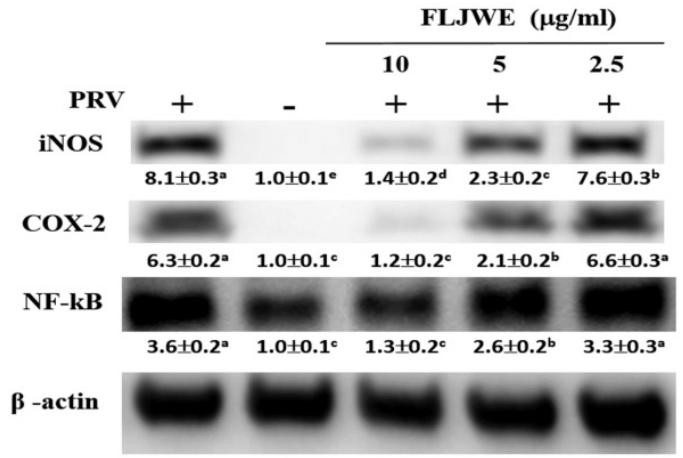

Figure 4. Effect of the FLJWE on iNOS, COX-2, and NF-KB expression in PRV-infected RAW264.7 cells. RAW264.7 cells were not treated or pretreated with various concentrations of the FLJWE $(2.5,5$, and $10 \mu \mathrm{g} / \mathrm{mL})$ for $1.5 \mathrm{~h}$ and then infected or not infected (control) with PRV (at an $\mathrm{MOI}$ of 0.1 ) for $24 \mathrm{~h}$. All proteins were subjected to $10 \%$ SDS-PAGE followed by Western blotting with iNOS, COX-2, $N F-K B$, and $\beta$-actin antibodies. Results are presented as mean $\pm S D(n=3)$. Mean values with different letters are significantly different $(p<0.05)$

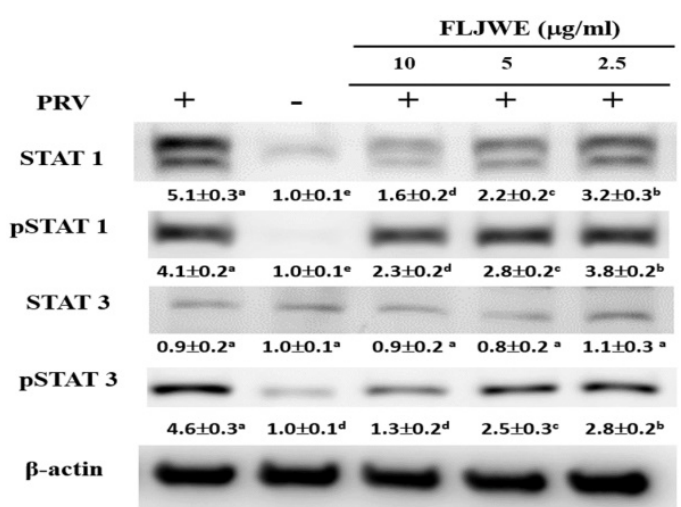

Figure 5. Effect of FLJWE on the expression of the STAT1/3 pathway in PRV-infected RAW264.7 cells. RAW264.7 cells were not treated or pretreated with various concentrations of the FLJWE $(2.5,5$, and $10 \mu \mathrm{g} / \mathrm{mL})$ for $1.5 \mathrm{~h}$ and then infected (control) or not infected with PRV (at an MOI of 0.1 ) for $24 \mathrm{~h}$. All proteins were subjected to $10 \%$ SDS-PAGE followed by Western blotting with STAT1/3, PSTAT1/3, and $\beta$-actin antibodies. Results are presented as mean $\pm S D(n=3)$. Mean values with different letters are significantly different $(p<0.05)$.

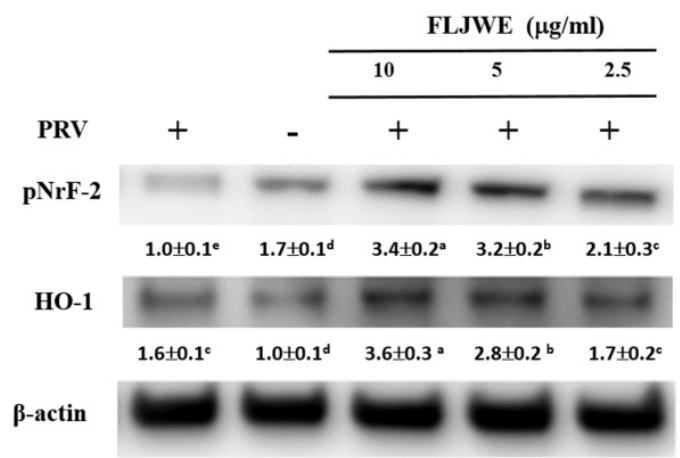

Figure 6. Effect of FLJWE on the expression of HO-1 and pNrf2 in PRV-infected RAW264.7 cells. RAW264.7 cells were not treated or pretreated with various concentrations of the FLJWE $(2.5,5$, and $10 \mu \mathrm{g} / \mathrm{mL})$ for $1.5 \mathrm{~h}$ and then infected or not infected with PRV (at an MOI of 0.1 ) for $24 \mathrm{~h}$. Total protein was subjected to $10 \%$ SDS-PAGE followed by Western blotting with $\mathrm{HO}-1, \mathrm{pNrf2}$, and $\beta$-actin antibodies. Results are presented as mean $\pm S D(n=3)$. Mean values with different letters are significantly different $(p<0.05)$.
Effect of FLJWE and comparison of the effects of chlorogenic acid and luteolin on NF- KB, COX-2, and iNOS expression in PRV-infected RAW264.7 cells

We evaluated the regulation of iNOS, COX-2, and NF-kB expression by the FLJWE, chlorogenic acid, and luteolin in PRV-infected RAW264.7 cells. The results indicated that the FLJWE and luteolin, but not chlorogenic acid, strongly inhibited the expression of NF-KB, iNOS, and COX-2 in PRV-infected RAW264.7 cells (Fig. 8).

Our previous studies have reported that FLJWE could induce HO-1 expression. Thus, we subsequently compared the regulatory effects of FLJWE, chlorogenic acid, and luteolin on HO-1 expression in infected RAW264.7 cells. Our results indicated that FLJWE and luteolin strongly upregulated $\mathrm{HO}-1$ expression in these cells. However, chlorogenic acid could only slightly induce HO-1 expression (Fig. 8B).

\section{Conclusions}

More than 212 chemical compounds have been isolated from FLJ, and most of these are phenolic acids (e.g., chlorogenic acid, isochlorogenic acid, caffeic acid, and hexadecanoic acid), essential oils, flavones (e.g., quercetin, luteolin, and hyperoside), iridoids, saponins, and trace minerals $[1,38,39,40]$. Zhang et al. (2011) evaluated the correlation between the antioxidative and anti-inflammatory activity of 14 Chinese medicinal plants by using water and ethanol extracts, and they reported that the high antioxidative activity of the extracts significantly contributed to the anti-inflammatory activity of these medicinal herbs. In addition, a study indicated that plant extracts containing high concentrations of phenolic acids and flavonoids exhibited substantial anti-inflammatory activity, resulting in high cell viability [22]. According to the results of the aforementioned studies, the antioxidative activity of herbs can be attributed to their total concentrations of flavonoids and phenolic acids. Many studies have reported that extracts exhibiting strong antioxidative activity significantly contributed to the anti-inflammatory activity of medicinal herbs. The results of this study demonstrated that the FLJWE contains many polyphenols, with chlorogenic acid and p-hydroxbenzoic acid being the major phenolic acids and epicatechin, luteolin, hesperidin, and morin being the major flavonoids (Fig. 1B).

The experimental results indicated that the FLJWE exerted an anti-inflammatory effect on virus-induced inflammation by inhibiting iNOS, COX-2, and NF-KB (Fig. 4) and cytokines such as TNF-a, MCP-1, RANTES, and IL-6 (Fig. 3). A previous 

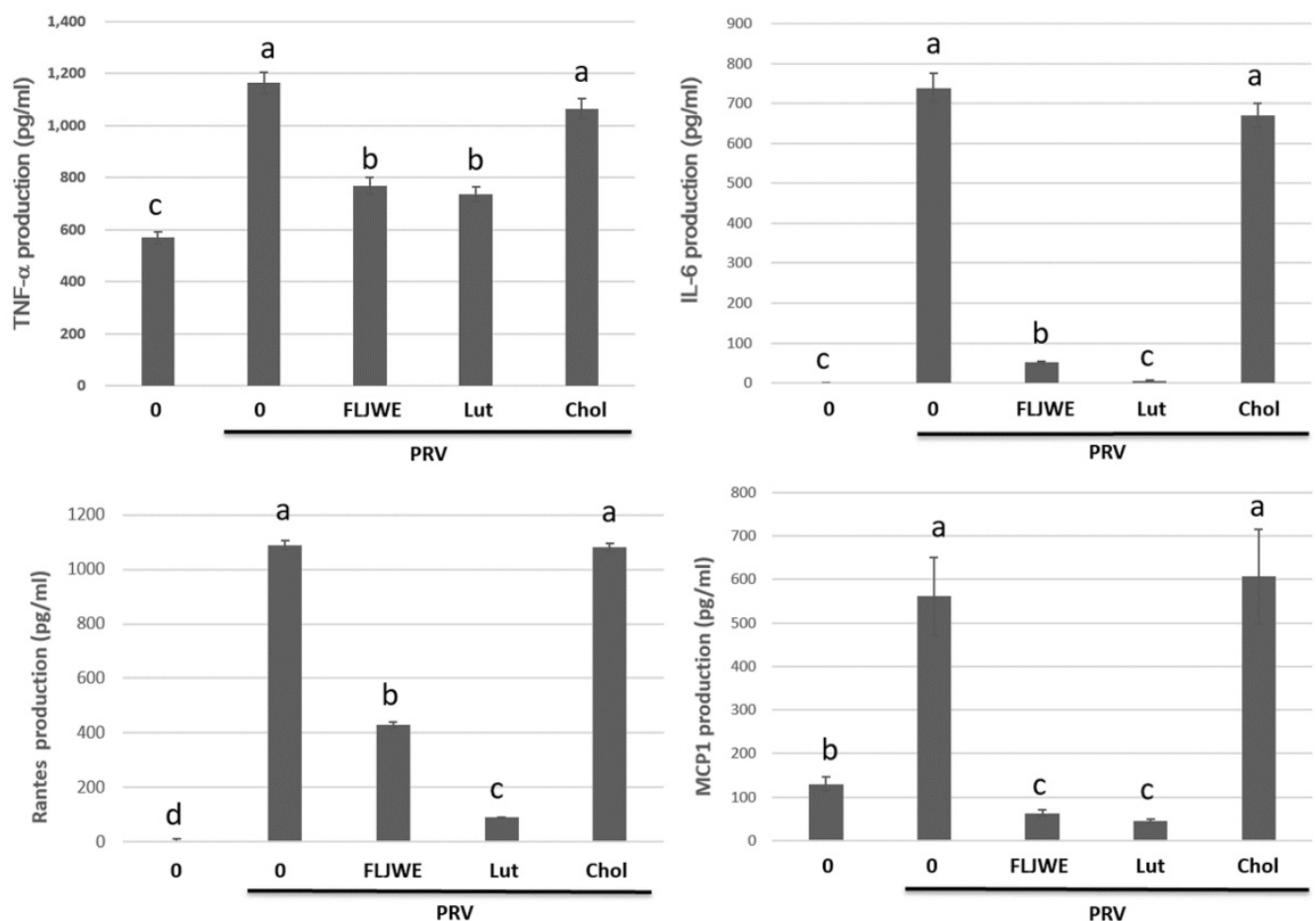

Figure 7. Effect of FLJWE, chlorogenic acid, and luteolin on inflammatory cytokines. RAW264.7 cells were not treated (control and PRV group) or pretreated with various concentrations of FLJWE, chlorogenic acid (Chol; $250 \mu \mathrm{M}$ ), or luteolin (Lut; $10 \mu \mathrm{M}$ ) for $1.5 \mathrm{~h}$ and then infected or uninfected (control) with PRV (at an MOI of 0.1 ). After $24 \mathrm{~h}$, the cultured medium was assayed to determine the level of IL-6, MCP-1, RANTES, and TNF-a, which were measured using an enzyme-linked immunosorbent assay. Results are presented as mean $\pm S D(n=3)$. Mean values with different letters are significantly different $(p<0.05)$.

study reported that FLJ could attenuate LPS-induced inflammatory effects through NF-kB and MAPK (Cheng et al., 2014) or IRAK-1/TAK1 and TBK1/IRF3 [41] signaling pathways in RAW 264.7 macrophages. However, whether FLJWE can inhibit STAT/NF-KB-related inflammation in macrophages (RAW264.7 cells) infected with PRV remains unclear. In this study, we found that FLJWE could reduce the secretion of inflammatory cytokines (Fig. 3), and this might have further suppressed the activation of STAT1 and STAT3 in PRV-induced RAW264.7 cells (Fig. 5). This result is consistent with that of a previous study that reported that luteolin inhibits the phosphorylation of STAT1 and STAT3 (Liu et al., 2016). Furthermore, the results of this study indicated that luteolin attenuated inflammatory responses by inducing Nrf2-mediated HO-1 expression and by suppressing the STAT1/3-dependent NF-KB pathway in PRV-infected RAW264.7 cells.

HO-1 has been reported to exert antiviral effects on herpes simplex virus type 2 [42], bovine viral diarrhea virus [43], and porcine reproductive and respiratory syndrome virus [44] in vitro. We examined the regulatory effect of the FLJWE on the HO-1/Nrf2 signaling pathway in PRV-infected RAW264.7 cells. Our data indicated that the FLJWE significantly upregulated the protein expression of $\mathrm{pNrf} 2$ and its downstream target gene HO-1 (Fig. 6). Our results indicated that FLJWE and luteolin strongly upregulated HO-1 expression in PRV-infected RAW264.7 cells. However, chlorogenic acid could only slightly induce HO-1 expression (Fig. 8B).

A.

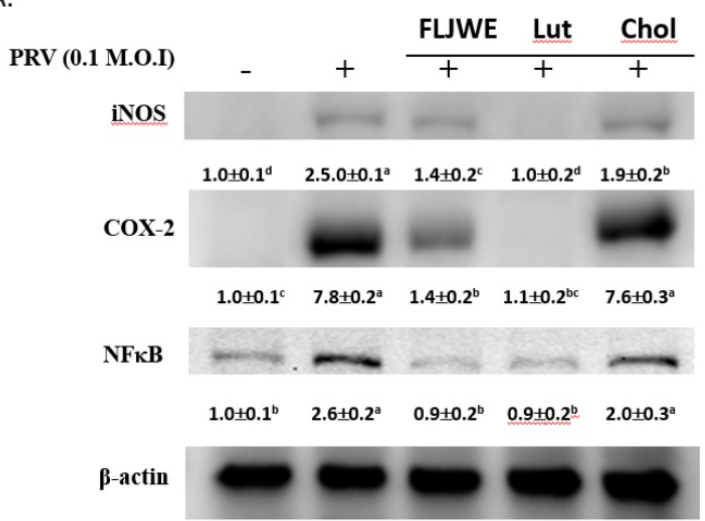

B.

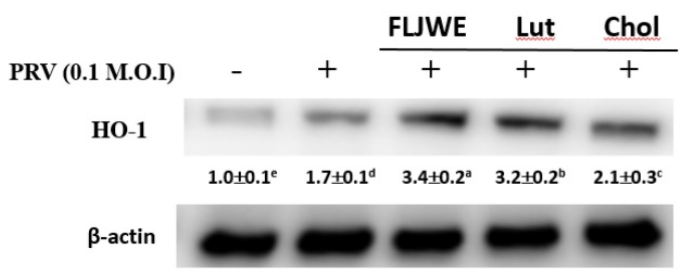

Figure 8. Comparison of the effects of FLJWE, chlorogenic acid, and luteolin on iNOS, NF-KB, and HO-1 expression in PRV-infected RAW264.7 cells. RAW264.7 cells were not treated (control and PRV group) or pretreated with various concentrations of FLJWE, chlorogenic acid (Chol; $250 \mu \mathrm{M}$ ), or luteolin (Lut; $10 \mu \mathrm{M}$ ) for $1.5 \mathrm{~h}$ and then infected or not infected (control) with PRV for $24 \mathrm{~h}$. All proteins were subjected to $10 \%$ SDS-PAGE followed by Western blotting with iNOS, NF-KB, $\mathrm{HO}-1$, and $\beta$-actin antibodies. Mean values with different letters are significantly different $(p<0.05)$. 


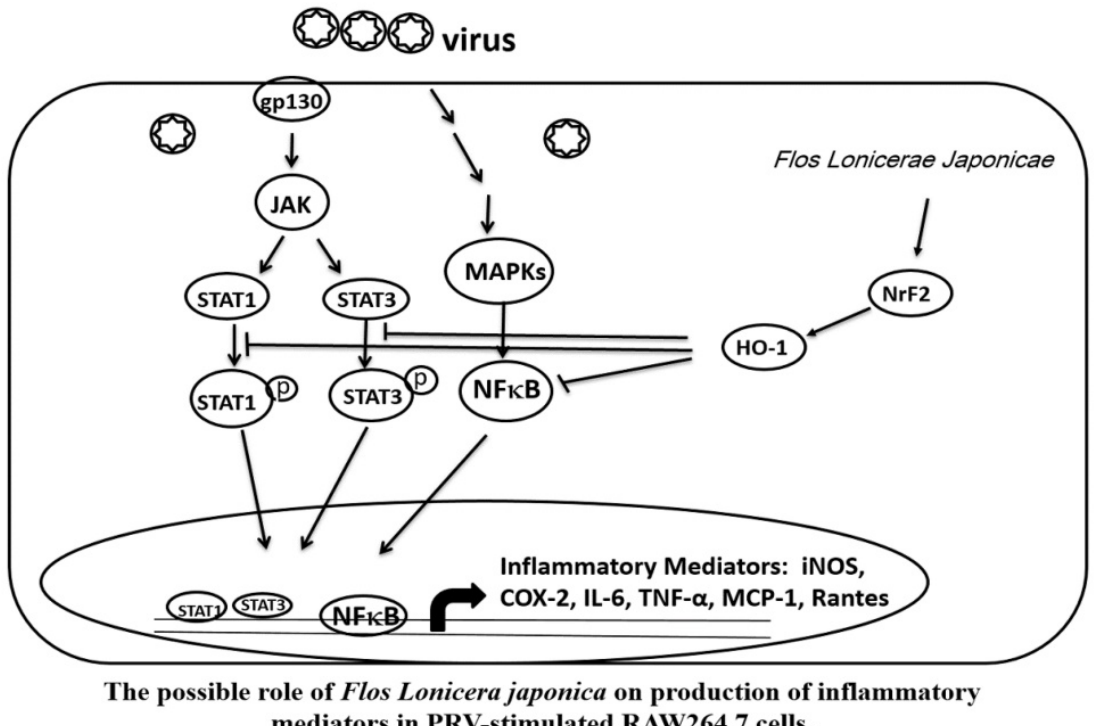

mediators in PRV-stimulated RAW264.7 cells.

Figure 9. Possible role of Flos Lonicerae Japonicae in the production of inflammatory mediators in PRV-stimulated RAW264.7 cells.

We observed that the major bioactive component of the FLJWE, chlorogenic acid, could not effectively inhibit virus-induced inflammatory responses (Figs. 7 and 8$)$. This result is consistent with that reported by Jin et al. (2006) who indicated that chlorogenic acid $(100 \mu \mathrm{g} / \mathrm{mL}$ of approximately $282 \mu \mathrm{M})$ did not exert an inhibitory effect on the levels of inflammatory mediators or the expression of COX-2 and iNOS in LPS-treated RAW 264.7 cells [39]. Shen et al. (2017) reported that up to $1 \mathrm{mM}$ chlorogenic acid could inhibit the LPS-induced expression of NO and IL-1 $\beta$ by inhibiting JAK2/STAT3 activation in RAW264.7 cells [45]. These findings indicate that luteolin is the active substance component present in the FLJWE that exerted the anti-inflammatory effect on PRV virusinduced inflammatory responses.

Taken together, our data indicate that the FLJWE exerted an antiviral effect on PRV-infected RAW264.7 cells by reducing the expression of proinflammatory mediators, such as COX-2 and iNOS and inflammatory cytokines, such as TNF-a, MCP-1, RANTES, and IL-6, through the suppression of the JAK/STAT1/3-dependent NF-kB pathway and the induction of HO-1 expression (Fig. 9). The findings of this study provide a pharmacological basis for using FLJWE, which contains many phenolic acids and flavonoids, as an antiviral and anti-inflammatory agent.

\section{Acknowledgements}

The authors thank the Ministry of Health and Welfare, Executive Yuan, Taiwan (ROC) (project number: CCMP102-RD-004), Chung Shan Medical University (Project: NCHU-CSMU-10708 and NCHU-CSMU-10613), and the Ministry of Science and Technology, Taiwan (project number: MOST-1052320-B-040-021 and MOST-106-2320-B-040-023-MY3) for financially supporting this research. The authors are grateful to Michael Burton of Asia University, Taiwan, for proofreading the manuscript.

\section{Ethics statement}

This research did not include any human subjects and animal experiments.

\section{Competing Interests}

The authors have declared that no competing interest exists.

\section{References}

1. $\mathrm{Li} \mathrm{Y,} \mathrm{Li} \mathrm{W}, \mathrm{Fu}$ C, et al. Lonicerae japonicae flos and Lonicerae flos: a systematic review of ethnopharmacology, phytochemistry and pharmacology. Phytochem Rev, 2019; 22: 1-61.

2. Wang T, Yang B, Guan Q, et al. Transcriptional regulation of Lonicera japonica Thunb. during flower development as revealed by comprehensive analysis of transcription factors. BMC Plant Biology. 2019, 19: 198.

3. Cheng BC, Yu H, Su T, et al. A herbal formula comprising Rosae Multiflorae Fructus and Lonicerae Japonicae Flos inhibits the production of inflammatory mediators and the IRAK-1/TAK1 and TBK1/IRF3 pathways in RAW 264.7 and THP-1 cells. J Ethnopharmacol, 2015; 174: 195-9.

4. Kao ST, Liu CJ, Yeh CC. Protective and immunomodulatory effect of flos Lonicerae japonicae by augmenting IL-10 expression in a murine model of acute lung inflammation. J Ethnopharmacol, 2015; 168: 108-115.

5. Shang X, Pan H, Li M, et al. Lonicera japonica Thunb.: ethnopharmacology, phytochemistry and pharmacology of an important traditional Chinese medicine. J Ethnopharmacol, 2011; 138: 1-21.

6. Park HS, Park KI, Lee DH, et al. Polyphenolic extract isolated from Korean Lonicera japonica Thunb. Induce G2/M cell cycle arrest and apoptosis in HepG2 cells: involvements of PI3K/Akt and MAPKs. Food Chem.Toxicol, 2012; 50: 2407-2416.

7. Lin HW, Chang TJ, Yang DJ, et al. Regulation of virus-induced inflammatory response by $\beta$-carotene in RAW264.7 cells. Food Chem. 2012; 134: 2169-2175.

8. Lin HW, Chen YC, Liu CW, et al. Regulation of virus-induced inflammatory response by Dunaliella salina alga extract in macrophages. Food Chem Toxicol. 2014; 71: 159-165.

9. Lin HW, Liu CW, Yang DJ, et al. Dunaliella salina alga extract inhibits IL-6, $\mathrm{NO}$ and ROS production by regulating NF-kB/JAK/STAT in virus infected RAW264.7 cells. J Food Drug Anal. 2017; 25: 908-918.

10. Liu CW, Lin HW, Yang DJ, et al. Luteolin inhibits viral-induced inflammatory response in RAW264.7 cells via suppression of STAT1/3 dependent NF-kB and activation of HO-1. Free Radic Biol Med. 2016; 95: 180-189. 
11. Chang $\mathrm{CH}$, Chang $\mathrm{YT}$, Tseng $\mathrm{TH}$ et al. Mulberry leaf extract inhibit hepatocellular carcinoma cell proliferation via depressing IL-6 and TNF- $\alpha$ derived from adipocyte. J Food Drug Anal, 2018; 26: 1024-1032.

12. Horng CT, Liu ZH, Huang YT, et al. Extract from Mulberry (Morus australis) leaf decelerate acetaminophen induced hepatic inflammation involving downregulation of myeloid differentiation factor 88 (MyD88) signals. J Food Drug Anal. 2017; 26: 862-871.

13. Lee SB, Lee WS, Shin JS, et al. Xanthotoxin suppresses LPS-induced expression of iNOS, COX-2, TNF- $\mathrm{a}$, and IL-6 via AP-1, NF- $\mathrm{kB}$, and JAK-STAT inactivation in RAW 264.7 macrophages. Int Immunopharmacol. 2017; 49: 21-29.

14. O'Shea JJ, Plenge R. JAK and STAT signaling molecules in immunoregulation and immune-mediated disease. Immunity 2012; 36: 542-550.

15. Kim SH, Park SY, Park YL, et al. Chlorogenic acid suppresses lipopolysaccharide induced nitric oxide and interleukin $1 \beta$ expression by inhibiting JAK2/STAT3 activation in RAW264.7 cells. Mol Med Rep. 2017; 16: 9224-9232.

16. Shin JS, Kang SY, Lee HH, et al. Patriscabrin F from the roots of Patrinia scabra attenuates LPS-induced inflammation by downregulating NF-kB, AP-1, IRF3, and STAT1/3 activation in RAW 264.7 macrophages. Phytomedicine, 2020; 68: 153167.

17. Li BZ, Guo B, Zhang HY, et al. Therapeutic potential of HO-1 in autoimmune diseases. Inflammation, 2014; 37: 1779-1788.

18. Lv J, Su W, Yu Q, et al. Heme oxygenase-1 protects airway epithelium against apoptosis by targeting the proinflammatory NLRP3-RXR axis in asthma. J. Biol. Chem, 2018; 293: 18454-18465.

19. Li Y, Kakkar R, Wang J. In vivo and in vitro Approach to Anti-arthritic and Anti-inflammatory Effect of Crocetin by Alteration of Nuclear Factor-E2related Factor 2/hem Oxygenase (HO)-1 and NF-kB Expression. Front Pharmacol, 2018; 9: 1341

20. Albarakati AJA, Baty RS, Aljoudi AM, et al. Luteolin protects against lead acetate-induced nephrotoxicity through antioxidant, anti-inflammatory, anti-apoptotic, and Nrf2/HO-1 signaling pathways. Mol Biol Rep, 2020; 47(4): 2591-2603.

21. Kitakaze T, Makiyama A, Yamashita Y, et al. Low dose of luteolin activates Nrf2 in the liver of mice at start of the active phase but not that of the inactive phase. PLoS One, 2020; 15(4): e0231403.

22. Diaz $\mathrm{P}$, Jeong SC, Lee $\mathrm{S}$, et al. Antioxidant and anti-inflammatory activities of selected medicinal plants and fungi containing phenolic and flavonoid compounds. Chin Med, 2012; 7(1): 26.

23. Moon JK, Shibamoto T. Antioxidant assays for plant and food components. J. Agric. Food Chem, 2009; 57: 1655-1666.

24. Ravipati AS, Zhang L, Koyyalamudi SR, et al. Antioxidant and antiinflammatory activities of selected Chinese medicinal plants and their relation with antioxidant content. BMC Complement Altern Med, 2012; 12: 173.

25. Zhang L, Ravipati AS, Koyyalamudi SR, et al. Antioxidant and anti-inflammatory activities of selected medicinal plants containing phenolicand flavonoid compounds. J Agric Food Chem, 2011; 59 (23): 12361-12367.

26. Yuan $Y$, Wang Z, Jiang C, et al. Exploiting genes and functional diversity of chlorogenic acid and luteolin biosyntheses in Lonicera japonica and their substitutes. Gene, 2014; 534(2): 408-416.

27. Zhang B, Nan TG, Xin J, et al. Development of a colloidal gold-based lateral flow dipstick immunoassay for rapid detection of chlorogenic acid and luteoloside in Flos Lonicerae Japonicae. J Pharm Biomed Anal, 2019; 170: 83-88.

28. Park E, Kum S, Wang C, et al. Anti-inflammatory activity of herbal medicines: inhibition of nitric oxide production and tumor necrosis factor-alpha secretion in an activated macrophage-like cell line. J Agr Food Chem, 2005; 33: 415-424.

29. Kitakaze, T., Makiyama, A., Yamashita, Y., \& Ashida, H. (2020). Low dose of luteolin activates Nrf2 in the liver of mice at start of the active phase but not that of the inactive phase. PLoS One. 2020; 15(4): 0231403.

30. Peairs A, Dai R, Gan L, et al. Epigallocatechin-3-gallate (EGCG) attenuates inflammation in MRL/lpr mouse mesangial cells. Cell Mol Immunol. 2010; 7(2): 123-132.

31. Selim NM, Elgazar AA, Abdel-Hamid NM, et al. Chrysophanol, Physcion, Hesperidin and Curcumin Modulate the Gene Expression of ProInflammatory Mediators Induced by LPS in HepG2: In Silico and Molecular Studies. Antioxidants (Basel), 2019; 8(9): 371

32. Wang J, Guo C, Wei Z, et al. Morin suppresses inflammatory cytokine expression by downregulation of nuclear factor- $\mathrm{kB}$ and mitogen-activated protein kinase (MAPK) signaling pathways in lipopolysaccharide-stimulated primary bovine mammary epithelial cells. J Dairy Sci, 2016; 99(4): 3016-3022.

33. Yan JJ, Du GH, Qin XM, et al. Baicalein attenuates the neuroinflammation in LPS-activated BV-2 microglial cells through suppression of pro-inflammatory cytokines, COX2/NF-KB expressions and regulation of metabolic abnormality. Int Immunopharmacol, 2019; 79: 106092

34. Zhao XL, Yu L, Zhang SD, et al. Cryptochlorogenic acid attenuates LPS-induced inflammatory response and oxidative stress via upregulation of the Nrf2/HO-1 signaling pathway in RAW 264.7 macrophages. Int Immunopharmacol, 2020; 83: 106436.

35. Waza AA, Hamid Z, Ali S, et al. A review on heme oxygenase-1 induction: Is it a necessary evil. Inflamm. Res, 2018; 67: 579-588.

36. Espinoza JA, León MA, Céspedes PF, et al. Heme Oxygenase-1 Modulates Human Respiratory Syncytial Virus Replication and Lung Pathogenesis during Infection. J. Immunol, 2017; 199: 212-223.
37. Huang $\mathrm{H}$, Falgout $\mathrm{B}$, Takeda $\mathrm{K}$, et al. Nrf2-dependent induction of innate host defense via heme oxygenase-1 inhibits Zika virus replication. Virology, 2017; 503:1-5.

38. Wang $\mathrm{H}, \mathrm{Li} \mathrm{Y}$, Wang $\mathrm{S}$, et al. Comparative transcriptomic analyses of chlorogenic acid and luteolosides biosynthesis pathways at different flowering stages of diploid and tetraploid Lonicera japonica. PeerJ, 2020, 8: e8690.

39. Jin $\mathrm{XH}$, Ohgami $\mathrm{K}$, Shiratori $\mathrm{K}$, et al. Effects of blue honeysuckle (Lonicera caerulea L.) extract on lipopolysaccharide-induced inflammation in vitro and in vivo. Exp Eye Res, 2006; 82: 860-867.

40. Zhou L, Zhang T, Lu B, et al. Lonicerae Japonicae Flos attenuates diabetic retinopathy by inhibiting retinal angiogenesis. J Ethnopharmacol, 2016; 189: $117-125$

41. Cheng BC, Yu H, Su T, et al. A herbal formula comprising Rosae Multiflorae Fructus and Lonicerae Japonicae Flos inhibits the production of inflammatory mediators and the IRAK-1/TAK1 and TBK1/IRF3 pathways in RAW 264.7 and THP-1 cells. J Ethnopharmacol, 2015; 174: 195-9.

42. Ibáñez FJ, Farías MA, Retamal-Díaz A, et al. Pharmacological Induction of Heme Oxygenase-1 Impairs Nuclear Accumulation of Herpes Simplex Virus Capsids upon Infection. Front Microbiol, 2017; 8: 2108.

43. Zhang $\mathrm{C}, \mathrm{Pu} \mathrm{F}$, Zhang $\mathrm{A}$, et al. Heme oxygenase-1 suppresses bovine viral diarrhoea virus replication in vitro. Sci. Rep. 2015; 5: 15575.

44. Xiao S, Zhang A, Zhang C. et al. Heme oxygenase-1 acts as antiviral factor for porcine reproductive and respiratory syndrome virus infection and over-expression inhibits virus replication in vitro. Antiviral Res, 2014; 110: 60-69.

45. Shen W, Qi R, Zhang J, et al. Chlorogenic acid inhibits LPS-induced microglial activation and improves survival of dopaminergic neurons. Brain Res Bull, 2012; 88: 487-494. 